\title{
Well-posedness and global existence for the Novikov equation
}

\author{
XiNGLONG WU AND ZhaOYANG YiN
}

\begin{abstract}
In this paper, we mainly study the Cauchy problem of the Novikov equation. We first establish the local well-posedness and give the precise blow-up scenario for the equation. Then we show that the equation has smooth solutions which exist globally in time. Finally we prove that peakon solutions to the equation are global weak solutions.
\end{abstract}

Mathematics Subject Classification (2010): 35G25 (primary); 35L05 (secondary).

\section{Introduction}

In this paper, we consider the Cauchy problem for the following partial differential equation (PDE)

$$
\begin{cases}u_{t}-u_{t x x}+4 u^{2} u_{x}= & 3 u u_{x} u_{x x}+u^{2} u_{x x x}, \\ & t>0, x \in \mathbb{R}, \\ u(0, x)=u_{0}(x), & x \in \mathbb{R} .\end{cases}
$$

Equation (1.1) arises as a zero curvature equation $F_{t}-G_{x}+[F, G]=0$, this being the compatibility condition for the linear system [13]

$$
\left\{\begin{array}{l}
\Psi_{x}=F \Psi, \\
\Psi_{t}=G \Psi,
\end{array}\right.
$$

This work was partially supported by NSFC (No. 10971235), RFDP (No. 200805580014) and the key project of Sun Yat-sen University. NCET-08-0579.

Received July 12, 2010; accepted in revised form March 22, 2011. 
where $y=u-u_{x x}$,

$$
F=\left(\begin{array}{ccc}
0 & y \lambda & 1 \\
0 & 0 & y \lambda \\
1 & 0 & 0
\end{array}\right), G=\left(\begin{array}{ccc}
\frac{1}{3 \lambda^{2}}-u u_{x} & \frac{u_{x}}{\lambda}-u^{2} y \lambda & u_{x}^{2} \\
\frac{u}{\lambda} & -\frac{2}{3 \lambda^{2}} & -\frac{u_{x}}{\lambda}-u^{2} y \lambda \\
-u^{2} & \frac{u}{\lambda} & \frac{1}{3 \lambda^{2}}+u u_{x}
\end{array}\right) .
$$

It was discovered very recently by Novikov in a symmetry classification of nonlocal PDEs with cubic nonlinearity [21]. The perturbative symmetry approach [19] yields necessary conditions for a PDE to admit infinitely many symmetries. Using the approach, Novikov is able to isolate the Equation (1.1) and finds its first few symmetries. He subsequently finds a scalar Lax pair for it, also proves that the equation is integrable. It is convenient to define a new dependent variable $y$, Equation (1.1) can be written as

$$
y_{t}+u^{2} y_{x}+3 u u_{x} y=0, \quad y=u-u_{x x} .
$$

Camassa and Holm [3] derived the equation

$$
y_{t}+u y_{x}+2 u_{x} y=0, \quad y=u-u_{x x}
$$

from an asymptotic approximation to the Hamiltonian for the Green-Naghdi equations in shallow water theory. To begin with, the Camassa-Holm equation approximates unidirectional fluid flow in Euler's equations at the next order beyond the KdV equation, it has a bi-Hamiltonian structure [12] and is completely integrable [6], and with a Lax pair based on a linear spectral problem of second order. Also, there are smooth soliton solutions of Equation (1.2) on a non-zero constant background [4]. The Camassa-Holm equation has attracted a lot of interest in the past seventeen years for various reasons.

One might wonder whether the Camassa-Holm equation is the only integrable PDEs of its kind, being a shallow water equation whose dispersionless version has weak solitons. Degasperis and Procesi used an asymptotic integrability approach to isolate integrable third order equations, discovered the Degasperis-Procesi equation

$$
y_{t}+u y_{x}+3 u_{x} y=0, \quad y=u-u_{x x} .
$$

The Degasperis-Procesi equation can be regarded as a model for nonlinear shallow water dynamics. Degasperis, Holm and Hone [9] prove the formal integrability of Equation (1.3) by constructing a Lax pair. They also show [9] that it has a biHamiltonian structure and an infinite sequence of conserved quantities, and admits exact peakon solutions.

Despite the form is similar to the Camassa-Holm equation, it should be emphasized that these two equation are truly different. One of the important features of Equation (1.3) is that it has not only peakon solitons [9], i.e. solutions 
at the form $u(t, x)=c e^{-|x-c t|}$ and periodic peakon solutions [23], but also shock peakons $[5,18]$ which are given by

$$
u(t, x)=-\frac{1}{t+k} \operatorname{sgn}(x) e^{-|x|}, \quad k>0
$$

and periodic shock peakons [11].

The Camassa-Holm equation has $n$-peakon solutions [2]

$$
u(t, x)=\sum_{j=1}^{n} p_{j}(t) \exp \left(-\left|x-q_{j}(t)\right|\right)
$$

where the position $q_{j}$ and amplitudes $p_{j}$ satisfy the system of ODEs

$$
\left\{\begin{array}{l}
\dot{q}_{j}=\sum_{j=1}^{n} p_{k} \exp \left(-\left|q_{j}-q_{k}\right|\right) \\
\dot{p}_{j}=p_{j} \sum_{k=1}^{n} p_{k} \operatorname{sgn}\left(q_{j}-q_{k}\right) \exp \left(-\left|q_{j}-q_{k}\right|\right)
\end{array}\right.
$$

here $j=1, \cdots, n$.

Analogous to the Camassa-Holm equation, the Novikov equation has a biHamiltonian structure and an infinite sequence of conserved quantities, and admits exact peakon solutions [13], i.e. solutions at the form

$$
u(t, x)= \pm \sqrt{c} e^{-\left|x-c t-x_{0}\right|}, \quad c>0, \quad x_{0} \text { constant. }
$$

Also Equation (1.1) has $n$-peakon solutions [13]

$$
u(t, x)=\sum_{j=1}^{n} p_{j}(t) \exp \left(-\left|x-q_{j}(t)\right|\right)
$$

where the position $q_{j}$ and amplitudes $p_{j}$ satisfy the system of ODEs

$$
\left\{\begin{array}{l}
\dot{q}_{j}=\sum_{k, l=1}^{n} p_{k} p_{l} \exp \left(-\left|q_{j}-q_{k}\right|-\left|q_{j}-q_{l}\right|\right), \\
\dot{p}_{j}=p_{j} \sum_{k, l=1}^{n} p_{k} q_{l} \operatorname{sgn}\left(q_{j}-q_{k}\right) \exp \left(-\left|q_{j}-q_{k}\right|-\left|q_{j}-q_{l}\right|\right),
\end{array}\right.
$$

here $j=1, \cdots, n$.

The remainder of the paper is organized as follows. In Section 2, we establish local well-posedness of Equation (1.1). In Section 3, we derive a precise blow-up scenario and present a global existence result of strong solutions to Equation (1.1). In Section 4, we prove that Equation (1.1) has weak solutions. 


\section{Local well-posedness}

In this section, we will apply Kato's theory to establish the local well-posedness for the Cauchy problem of Equation (1.1).

For convenience, we state Kato's theory in a form suitable for our purpose.

Consider the abstract quasi-linear evolution equation

$$
\frac{d u}{d t}+A(u) u=f(u), \quad t \geq 0, \quad u(0)=u_{0}
$$

Let $X$ and $Y$ be Hilbert spaces such that $Y$ is continuously and densely embedded in $X$ and let $S: Y \rightarrow X$ be a topological isomorphism. $L(Y, X)$ denotes the space of all bounded linear operators from $Y$ to $X(L(X)$, if $X=Y)$. Assume that:

(i) $A(u) \in L(Y, X)$ for $u \in Y$ with

$$
\|(A(y)-A(z)) w\|_{X} \leq a_{1}\|y-z\|_{X}\|w\|_{Y}, \quad y, z, w \in Y
$$

and $A(u) \in G(X, 1, \beta)$, i.e. $A(u)$ is quasi-m-accretive, uniformly on bounded sets in $Y$.

(ii) $S A(y) S^{-1}=A(y)+B(y)$, where $B(y) \in L(X)$ is bounded, uniformly on bounded sets in $Y$. Moreover,

$$
\|(B(y)-B(z)) w\|_{X} \leq a_{2}\|y-z\|_{Y}\|w\|_{X}, \quad y, z \in Y, w \in X .
$$

(iii) $f: Y \rightarrow Y$ extends to a map from $X$ into $X$. $f$ is bounded on bounded sets in $Y$, and satisfies

$$
\begin{array}{ll}
\|f(y)-f(z)\|_{Y} \leq a_{3}\|y-z\|_{Y} \quad y, z \in Y, \\
\|f(y)-f(z)\|_{X} \leq a_{4}\|y-z\|_{X} \quad y, z \in Y .
\end{array}
$$

Here $a_{1}, a_{2}, a_{3}, a_{4}$ depend only on $\max \left\{\|y\|_{Y},\|z\|_{Y}\right\}$.

Theorem 2.1 ([14, Kato]). Assume that (i), (ii), and (iii) hold. Given $v_{0} \in Y$, there is a $T>0$ depending only on $\left\|v_{0}\right\|_{Y}$ and a unique solution $v$ to Equation (2.1) such that

$$
v=v\left(., v_{0}\right) \in C([0, T) ; Y) \cap C^{1}([0, T) ; X) .
$$

Moreover, the map $v_{0} \rightarrow v$ is continuous from $Y$ to $C([0, T) ; Y) \cap C^{1}([0, T) ; X)$.

We provide now the framework in which we shall reformulate the Cauchy problem of Equation (1.1).

First, we introduce some notations. All spaces of functions are over $\mathbb{R}$ and for simplicity, we drop $\mathbb{R}$ in our notation of function spaces if there is no ambiguity. Additionally, if $A$ is an unbounded operator, $D(A)$ denotes the domain of the operator $A,[A, B]=A B-B A$ denotes the commutator of the linear operators $A$ 
and $B,\|\cdot\|_{X}$ denotes the norm of the Banach space $X$. For convenience, let $\|\cdot\|_{s}$ and $(\cdot, \cdot)_{s}$ denote the norm and the inner product of $H^{s}, s \in \mathbb{R}$, respectively, where $L^{r}, H^{s}$ denote $L^{r}(\mathbb{R}), H^{s}(\mathbb{R})$ spaces $, r \geq 1, s \in \mathbb{Z}$.

With $y=u-u_{x x}$, Equation (1.1) takes the form of a quasi-linear evolution equation of hyperbolic type

$$
\begin{cases}y_{t}+u^{2} y_{x}+3 u u_{x} y=0, & t>0, x \in \mathbb{R} \\ y(0, x)=u_{0}(x)-u_{x x}(0, x), & x \in \mathbb{R}\end{cases}
$$

Note that if $p(x)=\frac{1}{2} e^{-|x|}, x \in \mathbb{R}$, we have $\left(1-\partial_{x}^{2}\right)^{-1} f=p * f$ for all the $f \in L^{2}$, and $p * y=u$, here we denote by $*$ the convolution. Then we can rewrite Equation (2.2) as follows

$$
\begin{cases}u_{t}+u^{2} u_{x}+p *\left(3 u u_{x} u_{x x}+2 u_{x}^{3}+3 u^{2} u_{x}\right)=0, & t>0, x \in \mathbb{R} \\ u(0, x)=u_{0}(x), & x \in \mathbb{R}\end{cases}
$$

Or in the equivalent form

$$
\begin{cases}u_{t}+u^{2} u_{x}=-\left(1-\partial_{x}^{2}\right)^{-1}\left(\partial_{x}\left(\frac{3}{2} u u_{x}^{2}+u^{3}\right)+\frac{1}{2} u_{x}^{3}\right), & t>0, x \in \mathbb{R}, \\ u(0, x)=u_{0}(x), & x \in \mathbb{R} .\end{cases}
$$

Theorem 2.2. Assume that $u_{0} \in H^{s}, s>\frac{3}{2}$. Then there exists a unique solution $u$ to Equation (1.1) (or Equation (2.4)), and a $T=T\left(\left\|u_{0}\right\|_{s}\right)$ such that

$$
u=u\left(\cdot, u_{0}\right) \in C\left([0, T) ; H^{s}\right) \cap C^{1}\left([0, T) ; H^{s-1}\right) .
$$

Moreover, the solution depends continuously on the initial data, i.e. the mapping $u_{0} \rightarrow u\left(\cdot, u_{0}\right): H^{s} \rightarrow C\left([0, T) ; H^{s}\right) \cap C^{1}\left([0, T) ; H^{s-1}\right)$ is continuous.

Set $A(u)=u^{2} \partial_{x}, f(u)=-\left(1-\partial_{x}^{2}\right)^{-1}\left(3 u u_{x} u_{x x}+2 u_{x}^{3}+3 u^{2} u_{x}\right)=-(1-$ $\left.\partial_{x}^{2}\right)^{-1}\left(\partial_{x}\left(\frac{3}{2} u u_{x}^{2}+u^{3}\right)+\frac{1}{2} u_{x}^{3}\right), X=H^{s-1}, Y=H^{s}$ and $S=\Lambda=\left(1-\partial_{x}^{2}\right)^{\frac{1}{2}}$. Obviously, $S$ is an isomorphism of $H^{s}$ onto $H^{s-1}$. In order to prove Theorem 2.2 , in view of Theorem 2.1 , we only need to prove that $A(u)$ and $f(u)$ satisfy the conditions (i), (ii) and (iii).

We first give the following four useful lemmas.

Lemma 2.3 ([15]). Let $f \in H^{s}, s>\frac{3}{2}$. Then

$$
\left\|\Lambda^{-r}\left[\Lambda^{r+k+1}, N_{f}\right] \Lambda^{-k}\right\|_{L\left(L^{2}\right)} \leq c\|f\|_{s}, \quad|r|,|k| \leq s-1,
$$

where $N_{f}$ is the operator of multiplication by $f, c$ is a constant depending only on $r, k$. 
Lemma 2.4 ([14]). Let $r$, $t$ be real constant such that $-r<t \leq r$. Then

$$
\begin{gathered}
\|f g\|_{t} \leq c\|f\|_{r}\|g\|_{t}, \quad \text { if } \quad r>\frac{1}{2}, \\
\|f g\|_{r+t-\frac{1}{2}} \leq c\|f\|_{r}\|g\|_{t}, \quad \text { if } \quad r<\frac{1}{2},
\end{gathered}
$$

where $c$ is a positive constant depending only on $r, t$.

Lemma 2.5 ([20]). Let $X$ and $Y$ be two Banach spaces and $Y$ be continuously and densely embedded in $X$. Let $-A$ be the infinitesimal generator of the $C_{0}$-semigroup $T(t)$ on $X$ and $S$ be an isomorphism from $Y$ onto $X . Y$ is $-A$-admissible (i.e. $T(t) Y \subset Y, \forall t \geq 0$, and the restriction of $T(t)$ to $Y$ is a $C_{0}$-semigroup on $Y$ ) if and only if $-A_{1}=-S A S^{-1}$ is the infinitesimal generator of the $C_{0}$-semigroup $T_{1}(t)=S T(t) S^{-1}$ on $X$. Moreover, if $Y$ is $-A$-admissible, then the part of $-A$ in $Y$ is the infinitesimal generator of the restriction of $T(t)$ to $Y$.

Lemma $2.6([16])$. Let $g \in C^{k}(\mathbb{R}, \mathbb{R})$ and $g(0)=0$. Then

$$
\|g(u)\|_{r} \leq \tilde{g}\left(\|u\|_{r}\right), \quad \frac{1}{2}<r \leq k
$$

Moreover, if $g \in C^{\infty}(\mathbb{R}, \mathbb{R})$ with $g(0)=0$, then

$$
\|g(u)\|_{r} \leq \tilde{g}\left(\|u\|_{r}\right), \quad r>\frac{1}{2},
$$

here $\tilde{g}$ is a monotone increasing function depending only on the $g$ function.

We break the argument into several lemmas.

Lemma 2.7. The operator $A(u)=u^{2} \partial_{x}$, with $u \in H^{s}, s>\frac{3}{2}$, belongs to $G\left(L^{2}, 1, \beta\right)$.

Proof. Since $L^{2}$ ia a Hilbert space, we have $A(u) \in G\left(L^{2}, 1, \beta\right)$ for some real number $\beta$ if and only if the following conditions hold [16]:

(a) $(A(u) y, y)_{0} \geq-\beta\|y\|_{0}^{2}$.

(b) The range of $\lambda I+A$ is all of $X$, for some (or all) $\lambda>\beta$.

We first prove (a). Since $u \in H^{s}, s>\frac{3}{2}, u$ and $u_{x}$ belong to $L^{\infty}$. Note that $\|u\|_{L^{\infty}},\left\|u_{x}\right\|_{L^{\infty}} \leq\|u\|_{s}$. Thus

$$
\begin{aligned}
\left|(A(u) y, y)_{0}\right| & =\left|\left(u^{2} \partial_{x} y, y\right)_{0}\right|=\left|\frac{1}{2}\left(u u_{x} y, y\right)_{0}\right| \\
& \leq \frac{1}{2}\|u\|_{L^{\infty}}\left\|u_{x}\right\|_{L^{\infty}}\|y\|_{0}^{2} \leq c\|u\|_{S}^{2}\|y\|_{0}^{2} .
\end{aligned}
$$

Setting $\beta=c\|u\|_{s}^{2}$, we have $(A(u) y, y)_{0} \geq-\beta\|y\|_{0}^{2}$. 
Next, we prove (b). Because $A(u)$ is a closed operator and satisfies (a), $(\lambda I+$ $A)$ has closed range in $L^{2}$ for all $\lambda>\beta$. Therefore, it suffices to show that $(\lambda I+A)$ has dense range in $L^{2}$ for all $\lambda>\beta$.

Given $u \in H^{s}, s>\frac{3}{2}$, and $y \in L^{2}$, we have the generalized Leibnitz formula

$$
\partial_{x}\left(u^{2} y\right)=2 u u_{x} y+u^{2} \partial_{x} y \quad \text { in } \quad H^{-1} .
$$

Since $u, u_{x} \in L^{\infty}$, we obtain

$$
\begin{aligned}
D(A) & =D\left(u^{2} \partial_{x}\right)=\left\{y \in L^{2}, u^{2} \partial_{x} y \in L^{2}\right\} \\
& =\left\{z \in L^{2},-\partial_{x}\left(u^{2} z\right) \in L^{2}\right\}=D\left(\left(u^{2} \partial_{x}\right)^{*}\right)=D\left(A^{*}\right) .
\end{aligned}
$$

Assume that the range of $(A+\lambda)$ is not all of $L^{2}$. Then there exists $z \in L^{2}, z \neq 0$ such that $((\lambda I+A) y, z)_{0}=0$ for all $y \in D(A)$. Since $H^{1} \subset D(A), D(A)$ is dense in $L^{2}$. Hence it follows that $z \in D\left(A^{*}\right)$ and $\lambda z+A^{*} z=0$ in $L^{2}$. Since $D(A)=D\left(A^{*}\right)$, multiplying by $z$ and integrating by parts, we obtain

$$
0=\left(\left(\lambda I+A^{*}\right) z, z\right)_{0}=(\lambda z, z)+(z, A z) \mid \geq(\lambda-\beta)\|z\|_{0}^{2}, \quad \forall \lambda>\beta,
$$

and thus $z=0$, which contradicts our assumption $z \neq 0$. This completes the proof of Lemma 2.7.

Lemma 2.8. The operator $A(u)=u^{2} \partial_{x}$, with $u \in H^{s}, s>\frac{3}{2}$, belongs to $G\left(H^{s-1}, 1, \beta\right)$.

Proof. Due to $H^{s-1}$ being Hilbert space, $A(u)$ belongs to $G\left(H^{s-1}, 1, \beta\right)$ for some real number $\beta$ if and only if the following conditions hold [14].

(a) $(A(u) y, y)_{s-1} \geq-\beta\|y\|_{s-1}^{2}$.

(b) $-A(u)$ is the infinitesimal generator of a $C_{0}$-semigroup on $H^{s-1}$.

First, let us prove (a). Due to $u \in H^{s}, s>\frac{3}{2}$, so $u$ and $u_{x}$ belong to $L^{\infty}$ and $\|u\|_{L^{\infty}},\left\|u_{x}\right\|_{L^{\infty}} \leq\|u\|_{s}$. Note that

$$
\begin{aligned}
\Lambda^{s-1}(A(u) y) & =\left[\Lambda^{s-1}, u^{2}\right] \partial_{x} y+u^{2} \Lambda^{s-1}\left(\partial_{x} y\right) \\
& =\left[\Lambda^{s-1}, u^{2}\right] \partial_{x} y+u^{2} \partial_{x} \Lambda^{s-1} y
\end{aligned}
$$

Then we have

$$
\begin{aligned}
(A(u) y, y)_{s-1} & =\left(\Lambda^{s-1}\left(u^{2} \partial_{x} y\right), \Lambda^{s-1} y\right)_{0} \\
& =\left(\left[\Lambda^{s-1}, u^{2}\right] \partial_{x} y, \Lambda^{s-1} y\right)_{0}-\left(u u_{x} \Lambda^{s-1} y, \Lambda^{s-1} y\right)_{0} \\
& \leq\left\|\left[\Lambda^{s-1}, u^{2}\right] \Lambda^{2-s}\right\|_{L\left(L^{2}\right)}\left\|\Lambda^{s-1} y\right\|_{0}^{2}+\left\|u u_{x}\right\|_{L^{\infty}}\left\|\Lambda^{s-1} y\right\|_{0}^{2} \\
& \leq\left(c\|u\|_{s}+\|u\|_{s}^{2}\right)\|y\|_{s-1}^{2},
\end{aligned}
$$


here we applied Lemma 2.3 with $r=0, k=s-2$. Letting $\beta=\left(c\|u\|_{s}+\|u\|_{s}^{2}\right)$, we have $(A(u) y, y)_{s-1} \geq-\beta\|y\|_{s-1}^{2}$.

Next, we prove (b). Let $S=\Lambda^{s-1}$. Note that $\mathrm{S}$ is an isomorphism of $H^{s-1}$ onto $L^{2}$ and $H^{s-1}$ is continuously and densely embedded in $L^{2}$ as $s>\frac{3}{2}$. Define

$$
A_{1}(u)=S A(u) S^{-1}=\Lambda^{s-1} A(u) \Lambda^{1-s}, \quad B_{1}(u)=A_{1}(u)-A(u) .
$$

Let $y \in L^{2}$ and $u \in H^{s-1}, s>\frac{3}{2}$. Then we have

$$
\begin{aligned}
\left\|B_{1}(u) y\right\|_{0} & =\left\|\left[\Lambda^{s-1}, u^{2} \partial_{x}\right] \Lambda^{1-s} y\right\|_{0} \\
& \leq\left\|\left[\Lambda^{s-1}, u^{2}\right] \Lambda^{2-s}\right\|_{L\left(L^{2}\right)}\left\|\Lambda^{-1} \partial_{x} y\right\|_{0} \\
& \leq c\|u\|_{s}\|y\|_{0},
\end{aligned}
$$

here we used Lemma 2.3 with $r=0, k=s-2$. Thus we obtain $B_{1}(u) \in L\left(L^{2}\right)$. Note that $A_{1}(u)=A(u)+B_{1}(u)$ and $A(u) \in G\left(L^{2}, 1, \beta\right)$ in Lemma 2.7. By a perturbation theorem of semigroups (cf. [20, Theorem 2.3, Section 5.2]), we obtain $A_{1}(u) \in G\left(L^{2}, 1, \beta_{1}\right)$. Using Lemma 2.5 with $X=L^{2}, Y=H^{s-1}, S=\Lambda^{s-1}$, we conclude that $H^{s-1}$ is $-A$-admissible. So $-A(u)$ is the infinitesimal generator of a $C_{0}$-semigroup on $H^{s-1}$. This completes the proof of Lemma 2.8.

Lemma 2.9. Let the operator $A(u)=u^{2} \partial_{x}$, with $u \in H^{s}, s>\frac{3}{2}$. Then operator $A(u) \in L\left(H^{s}, H^{s-1}\right)$. Moreover,

$$
\|(A(y)-A(z)) w\|_{s-1} \leq a_{1}\|y-z\|_{s-1}\|w\|_{s}, \quad y, z, w \in H^{s} .
$$

Proof. Let $y, z, w \in H^{s}, s>\frac{3}{2}$. Note that $H^{s-1}$ is a Banach algebra. Then we have

$$
\begin{aligned}
\|(A(y)-A(z)) w\|_{s-1} & \leq c\left\|y^{2}-z^{2}\right\|_{s-1}\left\|\partial_{x} w\right\|_{s-1} \\
& \leq c\|(y+z)(y-z)\|_{s-1}\|w\|_{s} \\
& \leq a_{1}\|y-z\|_{s-1}\|w\|_{s} .
\end{aligned}
$$

Take $z=0$ in the above inequality to obtain $A(u) \in L\left(H^{s}, H^{s-1}\right)$. This completes the proof of Lemma 2.9.

Lemma 2.10. The operator $B(u)=\left[\Lambda, u^{2} \partial_{x}\right] \Lambda^{-1} \in L\left(H^{s-1}\right)$, with $u \in H^{s}, s>$ $\frac{3}{2}$. Moreover,

$$
\|(B(y)-B(z)) w\|_{s-1} \leq a_{2}\|y-z\|_{s}\|w\|_{s-1} .
$$

Proof. Let $y, z \in H^{s}, w \in H^{s-1}, s>\frac{3}{2}$. Note that $H^{s}$ is a Banach algebra. Then

$$
\begin{aligned}
\|(B(y)-B(z)) w\|_{s-1} & =\left\|\Lambda^{s-1}\left[\Lambda,\left(y^{2}-z^{2}\right) \partial_{x}\right] \Lambda^{-1} w\right\|_{0} \\
& \leq\left\|\Lambda^{s-1}\left[\Lambda,\left(y^{2}-z^{2}\right)\right] \Lambda^{1-s}\right\|_{L\left(L^{2}\right)}\left\|\Lambda^{s-2} \partial_{x} w\right\|_{0} \\
& \leq a_{2}\|y-z\|_{s}\|w\|_{s-1},
\end{aligned}
$$

where we applied Lemma 2.3 with $r=1-s, k=s-1$. Take $z=0$ in the above inequality to obtain $B(u) \in L\left(H^{s-1}\right)$. This completes the proof of Lemma 2.10. 
Lemma 2.11. Let $f(u)=-\left(1-\partial_{x}^{2}\right)^{-1}\left(\partial_{x}\left(\frac{3}{2} u u_{x}^{2}+u^{3}\right)+\frac{1}{2} u_{x}^{3}\right)$. Then $f$ is bounded on bounded set in $H^{s}$ and satisfies for all $s \geq \frac{3}{2}$,

$$
\begin{gathered}
\|f(y)-f(z)\|_{s} \leq a_{3}\|y-z\|_{s}, \quad y, z \in H^{s}, \\
\|f(y)-f(z)\|_{s-1} \leq a_{4}\|y-z\|_{s-1}, \quad y, z \in H^{s} .
\end{gathered}
$$

Proof. Let $y, z, \in H^{s}, s>\frac{3}{2}$. Since $H^{s-1}$ is a Banach algebra, we have

$$
\begin{aligned}
\|f(y)-f(z)\|_{s} \leq & \left\|-\partial_{x}\left(1-\partial_{x}^{2}\right)^{-1}\left(\frac{3}{2} y y_{x}^{2}+y^{3}-\frac{3}{2} z z_{x}^{2}-z^{3}\right)\right\|_{s} \\
& +\left\|-\left(1-\partial_{x}^{2}\right)^{-1}\left(\frac{1}{2} y_{x}^{3}-\frac{1}{2} z_{x}^{3}\right)\right\|_{s} \\
& \leq \frac{3}{2}\left\|y y_{x}^{2}-z z_{x}^{2}\right\|_{s-1}+\left\|y^{3}-z^{3}\right\|_{s-1}+\frac{1}{2}\left\|y_{x}^{3}-z_{x}^{3}\right\|_{s-1} .
\end{aligned}
$$

In view of Lemma $2.6, u \rightarrow g(u)-g(0)$ is a $C^{\infty}$-map from $H^{s-1}$ to $H^{s-1}$, where $g(u)=u, u^{2}$. From the mean value theorem [10], we infer that there is a some $M>0$, depending only on $\max \left\{\|y\|_{s},\|z\|_{s}\right\}$, such that

$$
\|g(y)-g(z)\|_{s-1} \leq M\|y-z\|_{s-1} .
$$

Hence

$$
\begin{aligned}
\|f(y)-f(z)\|_{s} & \leq \frac{3}{2}\left\|(y-z) y_{x}^{2}\right\|_{s-1}+\frac{3}{2}\left\|z\left(y_{x}^{2}-z_{x}^{2}\right)\right\|_{s-1}+\frac{3 M}{2}\|y-z\|_{s} \\
& \leq c\|y-z\|_{s-1}+c\|y-z\|_{s}+\frac{3 M}{2}\|y-z\|_{s} \\
& \leq c\|y-z\|_{s} .
\end{aligned}
$$

Taking $z=0$ in the above inequality, we obtain that $f$ is bounded on bounded set in $H^{s}$.

Next, let $y, z \in H^{s}, s>\frac{3}{2}$. Since $H^{s-1}$ is a Banach algebra, we have

$$
\begin{aligned}
\|f(y)-f(z)\|_{s-1} & \leq\left\|-\partial_{x}\left(1-\partial_{x}^{2}\right)^{-1}\left(\frac{3}{2} y y_{x}^{2}+y^{3}-\frac{3}{2} z z_{x}^{2}-z^{3}\right)\right\|_{s-1} \\
& +\left\|-\left(1-\partial_{x}^{2}\right)^{-1}\left(\frac{1}{2} y_{x}^{3}-\frac{1}{2} z_{x}^{3}\right)\right\|_{s-1} \\
& \leq \frac{3}{2}\left\|y y_{x}^{2}-z z_{x}^{2}\right\|_{s-2}+\left\|y^{3}-z^{3}\right\|_{s-1}+\frac{1}{2}\left\|y_{x}^{3}-z_{x}^{3}\right\|_{s-2 .} . \\
& \leq \frac{3}{2}\left\|(y-z) y_{x}^{2}\right\|_{s-2}+\frac{3}{2}\left\|z\left(y_{x}^{2}-z_{x}^{2}\right)\right\|_{s-2}+\frac{3 M}{2}\|y-z\|_{s-1} \\
& \leq c\|y-z\|_{s-1}+c\|y-z\|_{s-1}+\frac{3 M}{2}\|y-z\|_{s-1} \\
& \leq c\|y-z\|_{s-1} .
\end{aligned}
$$

This completes the proof of Lemma 2.11. 
Proof of Theorem 2.2. Combining Theorem 2.1 and Lemmas 2.8-2.11, we can get the statement of Theorem 2.2.

Theorem 2.12. Assume that $u_{0} \in H^{s}, s>\frac{3}{2}$. Then $T$ in Theorem 2.2 may be chosen independent of $s$ in the following sense. If $u=u\left(\cdot, u_{0}\right) \in C\left([0, T) ; H^{s}\right) \cap$ $C^{1}\left([0, T) ; H^{s-1}\right)$ to Equation (1.1) (or Equation (2.4)), and if $u_{0} \in H^{s_{1}}$ for some $s_{1} \neq s, s_{1}>\frac{3}{2}$, then $u \in C\left([0, T) ; H^{s_{1}}\right) \cap C^{1}\left([0, T) ; H^{s_{1}-1}\right)$ and with the same $T$. In particular, if $u_{0} \in H^{\infty}=\bigcap_{s \geq 0} H^{s}$, then $u \in C\left([0, T) ; H^{\infty}\right)$.

Proof. It suffices to consider the case $s_{1}>s$, since the case $s_{1}<s$ is obvious from uniqueness which is guaranteed by Theorem 2.2. In order to prove Theorem 2.12 for $s_{1}>s$, let us return to Equation (2.2). Set $y(t)=\Lambda^{2} u(t)$. Then we have

$$
\frac{d y}{d t}+A(t) y+B(t) y=0, \quad y(0)=\Lambda^{2} u(0)
$$

here $A(t) y=\partial_{x}\left(u^{2} y\right), B(t) y=u u_{x} y$.

Because $u \in C\left([0, T) ; H^{s}\right)$ and $u_{0} \in H^{s_{1}}$, we have $y \in C\left([0, T) ; H^{s-2}\right)$ and $y(0)=\Lambda^{2} u(0) \in C\left([0, T) ; H^{s_{1}-2}\right)$. It is our purpose to deduce $y \in C\left([0, T) ; H^{s_{1}-2}\right)$, which implies $u \in C\left([0, T) ; H^{s_{1}}\right)$. This will complete the proof of Theorem 2.12.

Since $u \in C\left([0, T) ; H^{s}\right), u_{x} \in H^{s-1}, H^{s-1}$ is a Banach algebra, we obtain $B(t) \in L\left(H^{s-1}\right)$.

To this end (see [15, Lemmas 3.1-3.3]), we first need to prove that the family $A(t)$ has a unique evolution operator $\{U(t, \tau)\}$ associated with the spaces $X=H^{h}$ and $Y=H^{k}$, where $-s \leq h \leq s-2,1-s \leq k \leq s-1$, and $k \geq h+1$. Therefore, according to the proof of [15, Lemma 2.1$]$, we need to verify the following three conditions.

(i) $A(t) \in G\left(H^{h}, 1, \beta\right), \quad \forall y \in H^{s}$.

(ii) $\Lambda^{h} \partial_{x}\left[\Lambda^{k-h}, u^{2}\right] \Lambda^{-k}$ is uniformly bounded on $L^{2}$.

(iii) $A(t) \in L\left(H^{k}, H^{h}\right)$ is strongly continuous in $t$.

Let us begin verify (i). Due to $H^{h}$ being a Hilbert space, $A(t) \in G\left(H^{h}, 1, \beta\right)$ [14] if and only if there is a real number $\beta$ such that

(a) $(A(t) y, y)_{h} \geq-\beta\|y\|_{h}^{2}$,

(b) $-A(t)$ is the infinitesimal generator of a $C_{0}$-semigroup on $H^{h}$.

First, we prove (a). Take $y \in H^{h}$. Note that

$$
\begin{aligned}
\Lambda^{h} \partial_{x}\left(u^{2} y\right) & =\Lambda^{h} \partial_{x}\left(-\left[\Lambda^{-h}, u^{2}\right] \Lambda^{h} y+\Lambda^{-h}\left(u^{2} \Lambda^{h} y\right)\right) \\
& =-\Lambda^{h} \partial_{x}\left[\Lambda^{-h}, u^{2}\right] \Lambda^{h} y+\partial_{x}\left(u^{2} \Lambda^{h} y\right) .
\end{aligned}
$$


Then we have

$$
\begin{aligned}
(A(t) y, y)_{h} & =-\left(\Lambda^{h} \partial_{x}\left[\Lambda^{-h}, u^{2}\right] \Lambda^{h} y, \Lambda^{h} y\right)_{0}+\left(\partial_{x}\left(u^{2} \Lambda^{h} y\right), \Lambda^{h} y\right)_{0} \\
& =\left(\Lambda^{h+1}\left[\Lambda^{-h}, u^{2}\right] \Lambda^{h} y, \partial_{x} \Lambda^{h-1} y\right)_{0}+\frac{1}{2}\left(u u_{x} \Lambda^{h} y, \Lambda^{h} y\right)_{0} \\
& \leq\left\|\Lambda^{h+1}\left[\Lambda^{-h}, u^{2}\right]\right\|_{L\left(L^{2}\right)}\left\|\Lambda^{h} y\right\|_{0}^{2}+\frac{1}{2}\left\|u u_{x}\right\|_{L^{\infty}}\left\|\Lambda^{h} y\right\|_{0}^{2} \\
& \leq\left(c\|u\|_{s}+c\|u\|_{s}^{2}\right)\|y\|_{h}^{2},
\end{aligned}
$$

where we applied Lemma 2.3 with $r=-(h+1), k=0$. Setting $\beta=\left(c\|u\|_{s}+\right.$ $\left.c\|u\|_{S}^{2}\right)$, we have $(A(t) y, y)_{h} \geq-\beta\|y\|_{h}^{2}$.

Secondly, we prove (b). Let $S=\Lambda^{s-1-h}$. Note that $S$ is an isomorphism of $H^{s-1}$ onto $H^{h}$ and $H^{s-1}$ is continuously and densely embedded in $H^{h}$ as $-s \leq$ $h \leq s-2$. Define

$$
\begin{aligned}
& A_{1}(t):=S A(t) S^{-1}=\Lambda^{s-1-h} A(t) \Lambda^{h+1-s}, \\
& B_{1}(t):=A_{1}(t)-A(t)=[S, A(t)] S^{-1} .
\end{aligned}
$$

Let $y \in H^{h}$ and $u \in H^{s}, s>\frac{3}{2}$. Then we have

$$
\begin{aligned}
\left\|B_{1}(t) y\right\|_{h} & =\left\|\Lambda^{h} \partial_{x}\left[\Lambda^{s-1-h}, u^{2}\right] \Lambda^{h+1-s} y\right\|_{0} \\
& \leq\left\|\Lambda^{h} \partial_{x}\left[\Lambda^{s-1-h}, u^{2}\right] \Lambda^{1-s}\right\|_{L\left(L^{2}\right)}\left\|\Lambda^{h} y\right\|_{0} \\
& \leq c\|u\|_{s}\|y\|_{h},
\end{aligned}
$$

where we applied Lemma 3.1 with $r=-(h+1), k=s-1$. Therefore, we obtain $B_{1}(t) \in L\left(H^{h}\right)$. Note that

$$
A(t) y=\partial_{x}\left(u^{2} y\right)=2 u u_{x} y+u^{2} \partial_{x} y \quad \text { and } \quad u_{x} \in L\left(H^{s-1}\right) .
$$

Applying Lemma 2.8 and a perturbation theorem for semigroups, we have $H^{s-1}$ is $-A(t)$-admissible. Then by applying Lemma 2.5 with $Y=H^{s-1}, X=H^{h}$ and $S=\Lambda^{s-1-h}$, we obtain that $-A_{1}(t)$ is the infinitesimal generator of a $C_{0^{-}}$ semigroup on $H^{h}$. Due to $A_{1}(t)=A(t)+B_{1}(t)$ and $B_{1}(t) \in L\left(H^{h}\right)$, by a perturbation theorem for semigroups, we have that $-A(t)$ is the infinitesimal generator of a $C_{0}$-semigroup on $H^{h}$. This proves (b).

Next, we verify (ii). Take $y \in L^{2}$. Then we have

$$
\left\|\Lambda^{h} \partial_{x}\left[\Lambda^{k-h}, u^{2}\right] \Lambda^{-k} y\right\|_{0} \leq c\|u\|_{s}\|y\|_{0},
$$

where we applied Lemma 2.3 with $r=-(h+1), k=k$.

Finally, we verify (iii). Take $y \in H^{k}$. Then

$$
\begin{aligned}
\left\|\partial_{x}\left(\left(u^{2}(t+\tau)-u^{2}(t)\right) y\right)\right\|_{h} & \leq\left\|\left(u^{2}(t+\tau)-u^{2}(t)\right) y\right\|_{h+1} \\
& \leq c\left\|u^{2}(t+\tau)-u^{2}(t)\right\|_{s-1}\|y\|_{h+1} \\
& \leq c\|u\|_{s}\|u(t+\tau)-u(t)\|_{s}\|y\|_{k},
\end{aligned}
$$


where we applied Lemma 2.4 with $r=s-1, t=h+1$. By the continuity of $u$, we prove (iii). Thus the above three conditions imply the existence and uniqueness of evolution operator $U(t, \tau)$ for the family $A(t)$. In particular $U(t, \tau)$ maps $H^{r}$ into itself for $-s \leq r \leq s-1$.

Next, we choose $Y=H^{s-2}$ and $X=H^{s-3}$. Note that

$$
y \in C\left([0, T) ; H^{s-2}\right) \cap C^{1}\left([0, T) ; H^{s-3}\right) .
$$

By the properties of evolution operator $U(t, \tau)$, we can obtain

$$
\frac{d}{d \tau}(U(t, \tau) y(\tau))=U(t, \tau)(-B(\tau) y(\tau)) .
$$

Integrating the above equality in $\tau \in[0, t]$, we obtain

$$
y(t)=U(t, 0) y(0)-\int_{0}^{t} U(t, \tau) B(\tau) y(\tau) d \tau .
$$

If $s<s_{1} \leq s+1$, then we have $B(t) \in L\left(H^{s_{1}-2}\right)$ is strongly continuous in [0,t), and $H^{s-1} H^{s_{1}-2} \subset H^{s_{1}-2}$ as $s-1>\frac{1}{2}$. Due to $-s<s-2<s_{1}-2 \leq s-1$, the family $\{U(t, \tau)\}$ is strongly continuous on $H^{s_{1}-2}$ to itself. Note that $y(0) \in$ $H^{s_{1}-2}$. We regard Equation (2.6) as an integral equation of Volterra type which can be solved for $y$ by successive approximation. Then the result of Theorem 2.12 is obtained.

If $s_{1}>s+1$, we obtain the result of Theorem 2.12 by repeated application of the above argument. This completes the proof of Theorem 2.12.

\section{Blow-up scenario and global existence}

In this section, we will begin deriving a conservation law for strong solutions to Equation (2.3). Using this conservation law, we obtain blow-up scenario. Then we establish a global existence theorem.

At first, we give the following useful lemmas.

Lemma 3.1 ([17]). Assume that $s>0$. Then we have

$$
\left\|\left[\Lambda^{s}, g\right] f\right\|_{L^{2}} \leq c\left(\left\|\partial_{x} g\right\|_{L^{\infty}}\left\|\Lambda^{s-1} f\right\|_{L^{2}}+\left\|\Lambda^{s} g\right\|_{L^{2}}\|f\|_{L^{\infty}}\right),
$$

where $c$ is constant depending only on $s$, and $f, g_{x} \in L^{\infty} \cap H^{s-1}$.

Lemma $3.2([8])$. Assume that $F \in C^{m+2}(\mathbb{R})$ with $F(0)=0$. Then for every $\frac{1}{2}<s \leq m$, we have

$$
\|F(u)\|_{s} \leq \tilde{F}\left(\|u\|_{L^{\infty}}\right)\|u\|_{s}, \quad u \in H^{s},
$$

where $\tilde{F}$ is a monotone increasing function depending only on $F$ and $s$. 
Lemma 3.3 ([17]). Assume that $s>0$. Then $H^{s} \cap L^{\infty}$ is an algebra. Moreover

$$
\|f g\|_{s} \leq c\left(\|f\|_{L^{\infty}}\|g\|_{s}+\|f\|_{s}\|g\|_{L^{\infty}}\right)
$$

where $c$ is a constant depending only on $s$, and $f, g \in L^{\infty} \cap H^{s}$.

Lemma 3.4 ([7]). Let $T>0$ and $u \in C^{1}\left([0, T) ; H^{2}\right)$. Then for every $t \in[0, T)$, there exist at least one pair points $\xi(t), \zeta(t) \in \mathbb{R}$, such that

$$
m(t)=\inf _{x \in \mathbb{R}} u(t, x)=u(t, \xi(t)), \quad M(t)=\sup _{x \in \mathbb{R}} u(t, x)=u(t, \zeta(t))
$$

and $m(t), M(t)$ are absolutely continuous in $[0, T)$. Moreover,

$$
\frac{d m(t)}{d t}=u_{t}(t, \xi(t)), \quad \frac{d M(t)}{d t}=u_{t}(t, \zeta(t)), \quad \text { a.e. } \quad \text { on } \quad[0, T) .
$$

Lemma 3.5. Let $u_{0} \in H^{s}, s>\frac{3}{2}$. Then as long as the solution $u(t, x)$ to Equation (1.1) given by Theorem 2.2 exists, we have

$$
\int_{\mathbb{R}}\left(u^{2}(t, x)+u_{x}^{2}(t, x)\right) d x=\int_{\mathbb{R}}\left(u_{0}^{2}+u_{0, x}^{2}\right) d x,
$$

where $u_{0}=u(0, x), u_{0, x}=u_{x}(0, x)$. Moreover, we have

$$
|u(t, x)| \leq \frac{\sqrt{2}}{2}\left\|u_{0}\right\|_{1}
$$

Proof. Applying Theorem 2.12 and a simple density argument, it suffices to consider $s=3$. Multiply Equation (1.1) by $u$, we have

$$
u u_{t}-u u_{t x x}+4 u^{3} u_{x}=3 u^{2} u_{x} u_{x x}+u^{3} u_{x x x} .
$$

Integrating by parts on $\mathbb{R}$,

$$
\begin{aligned}
\frac{1}{2} \frac{d}{d t} \int_{\mathbb{R}}\left(u^{2}+u_{x}^{2}\right) d x & =\int_{\mathbb{R}}\left(-4 u^{3} u_{x}+3 u^{2} u_{x} u_{x x}+u^{3} u_{x x x}\right) d x \\
& =\int_{\mathbb{R}}\left(3 u^{2} u_{x} u_{x x}-3 u^{2} u_{x} u_{x x}\right) d x \\
& =0 .
\end{aligned}
$$

Thus we deduce that

$$
\int_{\mathbb{R}}\left(u^{2}(t, x)+u_{x}^{2}(t, x)\right) d x=\int_{\mathbb{R}}\left(u_{0}^{2}+u_{0, x}^{2}\right) d x .
$$


In view of the above conservation law, we have

$$
\begin{aligned}
\sqrt{2}|u(t, x)| & =\left(\int_{-\infty}^{x} 2 u u_{x} d x-\int_{x}^{\infty} 2 u u_{x} d x\right)^{\frac{1}{2}} \\
& \leq\left(\int_{\mathbb{R}} 2\left|u u_{x}\right| d x\right)^{\frac{1}{2}} \leq\left(\int_{\mathbb{R}}\left(u^{2}+u_{x}^{2}\right) d x\right)^{\frac{1}{2}} \\
& =\left(\int_{\mathbb{R}}\left(u_{0}^{2}+u_{0, x}^{2}\right) d x\right)^{\frac{1}{2}}=\left\|u_{0}\right\|_{1} .
\end{aligned}
$$

This completes the proof of Lemma 3.5.

Theorem 3.6. Let $u_{0} \in H^{r}, r>\frac{3}{2}$. If $T$ is the maximal existence time of corresponding solution of the initial data $u_{0}$, then the $H^{r}$-norm of $u(t, x)$ to Equation (1.1) (or (2.4)) blows up on $[0, T)$ if and only if

$$
\varlimsup_{t \uparrow T}\left\|u_{x}(t, x)\right\|_{L^{\infty}}=\infty .
$$

Proof. Let $u(t, x)$ be the solution of Equation (1.1) with the initial data $u_{0} \in$ $H^{r}, r>\frac{3}{2}$, which is guaranteed by Theorem 2.2.

If $\varlimsup_{\lim _{t \uparrow T}}\left\|u_{x}(t, x)\right\|_{L^{\infty}}=\infty$, by Sobolev's embedding theorem, we obtain the solution $u(t, x)$ will blow up in finite time.

Next, applying the operator $\Lambda^{r}$ to Equation (2.4), multiplying by $\Lambda^{r} u$, and integrating by parts on $\mathbb{R}$, we have

$$
\frac{d}{d t}(u, u)_{r}=-2\left(u^{2} u_{x}, u\right)_{r}+2(f(u), u)_{r},
$$

where $f(u)=-\left(1-\partial_{x}^{2}\right)^{-1}\left(\partial_{x}\left(\frac{3}{2} u u_{x}^{2}+u^{3}\right)+\frac{1}{2} u_{x}^{3}\right)$.

Assume there exists a $M>0$, such that $\varlimsup_{\lim _{t \uparrow T}}\left\|u_{x}(t, x)\right\|_{L^{\infty}} \leq M$. Then we have

$$
\begin{aligned}
\left|\left(u^{2} u_{x}, u\right)_{r}\right| & =\left|\left(\Lambda^{r}\left(u^{2} u_{x}\right), \Lambda^{r} u\right)_{0}\right| \\
& =\left|\left(\left[\Lambda^{r}, u^{2}\right] u_{x}, \Lambda^{r} u\right)_{0}+\left(u^{2} \Lambda^{r} u_{x}, \Lambda^{r} u\right)_{0}\right| \\
& \leq\left\|\left[\Lambda^{r}, u^{2}\right] u_{x}\right\|_{L^{2}}\left\|\Lambda^{r} u\right\|_{L^{2}}+\left\|u u_{x}\right\|_{L^{\infty}}\left\|\Lambda^{r} u\right\|_{L^{2}}^{2} \\
& \leq c\left(\left\|\left(u^{2}\right)_{x}\right\|_{L^{\infty}}\left\|\Lambda^{r-1} u_{x}\right\|_{L^{2}}+\left\|\Lambda^{r} u^{2}\right\|_{L^{2}}\left\|u_{x}\right\|_{L^{\infty}}\right)\|u\|_{r} \\
& \quad+c M\|u\|_{r}^{2} \\
& \leq c\left(\left\|u u_{x}\right\|_{L^{\infty}}\|u\|_{r}+M\left\|u^{2}\right\|_{r}\right)\|u\|_{r}+c M\|u\|_{r}^{2} \\
& \leq c M\|u\|_{r}^{2} \leq c\|u\|_{r}^{2}
\end{aligned}
$$

where we applied Lemma 3.1 with $s=r$, Lemma 3.2 with $F(u)=u^{2}$ and $s=r$. 
Similarly, note that $H^{s}, s>\frac{1}{2}$ is a Banach algebra, it follows that

$$
\begin{aligned}
\left\|u u_{x}^{2}\right\|_{r-1} & =\left\|\left[\Lambda^{r-1}, u\right] u_{x}^{2}+u \Lambda^{r-1} u_{x}^{2}\right\|_{L^{2}} \\
& \leq c\left(\left\|\partial_{x} u\right\|_{L^{\infty}}\|\|_{L^{2}}+\left\|\Lambda^{r-1} u\right\|_{L^{2}}\left\|u_{x}^{2}\right\|_{L^{\infty}}\right) \\
& \leq c\left(M\left\|u_{x}^{2}\right\|_{r-1}+M^{2}\|u\|_{r-1}\right) \leq c\|u\|_{r} .
\end{aligned}
$$

On the other hand, we estimate the second term of the right hand side of Equation (3.1).

$$
\begin{aligned}
(f(u), u)_{r} & =\left(-\left(1-\partial_{x}^{2}\right)^{-1}\left(\partial_{x}\left(\frac{3}{2} u u_{x}^{2}+u^{3}\right)+\frac{1}{2} u_{x}^{3}\right), u\right)_{r} \\
& \leq c\|u\|_{r}\left(\frac{3}{2}\left\|u u_{x}^{2}+u^{3}\right\|_{r-1}+\frac{1}{2}\left\|u_{x}^{3}\right\|_{r-2}\right) \\
& \leq c\|u\|_{r}\left(\|u\|_{r}+\left\|u^{3}\right\|_{r-1}+\frac{1}{2}\left\|u_{x}^{3}\right\|_{r-1}\right) \\
& \leq c\|u\|_{r}^{2},
\end{aligned}
$$

where we applied Lemma 3.2 with $F(u)=u^{3}, u_{x}^{3}$ and $s=r-1$ and the above inequality.

From (3.1)-(3.3), we obtain

$$
\frac{d}{d t}\|u\|_{r}^{2} \leq c\|u\|_{r}^{2}
$$

Thus using Gronwall's inequality, we get

$$
\|u(t)\|_{r}^{2} \leq\left\|u_{0}\right\|_{r}^{2} \exp (c t) .
$$

This completes the proof of Theorem 3.6.

Consider the following differential equation

$$
\left\{\begin{array}{l}
q_{t}=u^{2}(t, q), t>0, x \in \mathbb{R}, \\
q(0, x)=x, \quad x \in \mathbb{R} .
\end{array}\right.
$$

Applying classical results in the theory of ordinary differential equations, one can obtain the following useful result on the above initial value problem.

Lemma 3.7. Let $u_{0} \in H^{s}, s \geq 3$, and $T$ be the maximal existence time of the corresponding solution $u(t, x)$ to Equation (2.3). Then Equation (3.4) has a unique solution $q \in C^{1}([0, T) \times \mathbb{R}, \mathbb{R})$. Moreover, the map $q(t, \cdot)$ is an increasing diffeomorphism of $\mathbb{R}$ with

$$
q_{x}=\exp \left(\int_{0}^{t} 2 u u_{x}(s, q(s, x)) d s\right), \quad \forall(t, x) \in[0, T) \times \mathbb{R} .
$$

Furthermore, setting $y=u-u_{x x}$, we obtain

$$
y(t, q) q_{x}^{\frac{3}{2}}(t, x)=y_{0}(x), \quad \forall(t, x) \in[0, T) \times \mathbb{R} .
$$


Proof. First, for fixed $x \in \mathbb{R}$ we deal with an ordinary differential equation. By the Sobolev's embedding theorem we have that $u \in C^{1}([0, T) \times \mathbb{R}, \mathbb{R})$. Therefore classical results in the theory of ordinary differential equations ([1]) yield the the first assertion. From Equation (3.4) we obtain

$$
\begin{cases}\frac{d}{d t} q_{x}=2 u(t, q) u_{x}(t, q) q_{x}, & t>0, x \in \mathbb{R}, \\ q_{x}(0, x)=1, & x \in \mathbb{R} .\end{cases}
$$

Thus the solution is given by

$$
q_{x}=\exp \left(\int_{0}^{t} 2 u u_{x}(s, q(s, x)) d s\right), \quad \forall(t, x) \in[0, T) \times \mathbb{R} .
$$

Applying Sobolev's embedding theorem, we can find a constant $K>0$ such that

$$
q_{x}(t, x) \geq e^{-t K}, \quad(t, x) \in[0, T) \times \mathbb{R} .
$$

Next, differentiating the left-hand side of the above equation with respect to the time variable $t$, and applying the relations, we have

$$
\begin{aligned}
\frac{d}{d t}\left(y(t, q) q_{x}^{\frac{3}{2}}(t, x)\right) & =\left(y_{t}+y_{x} q_{t}\right) q_{x}^{\frac{3}{2}}+\frac{3}{2} y q_{x}^{\frac{1}{2}} q_{x t} \\
& =\left(y_{t}+y_{x} q_{t}+3 u u_{x} y\right) q_{x}^{\frac{3}{2}} \\
& =\left(y_{t}+y_{x} u^{2}+3 u u_{x} y\right) q_{x}^{\frac{3}{2}} \\
& =0
\end{aligned}
$$

Thus we obtain

$$
y(t, q) q_{x}^{\frac{3}{2}}(t, x)=y_{0}(x), \quad \forall(t, x) \in[0, T) \times \mathbb{R} .
$$

This completes the proof of Lemma 3.7.

Next, in view of the blow-up scenario and Lemmas 3.5-3.7, we will establish the following global existence theorem.

Theorem 3.8. Assume that $u_{0} \in H^{s}, s>\frac{3}{2}$. If $y_{0}=u_{0}-u_{0 x x}$ doesn't change sign on $\mathbb{R}$, then Equation (2.3) has a global strong solution $u(t, x)$. Moreover, the solution $u(t, x)$ satisfies $\left|u_{x}(t, x)\right| \leq \frac{\sqrt{2}}{2}\left\|u_{0}\right\|_{1}$.

Proof. Applying Theorem 2.12 and a simple density argument, we only need to assume $s=3$. Let $T$ be the maximal existence time of the solution $u$ to Equation (2.3) with initial data $u_{0} \in H^{3}$. 
We first consider the case $y_{0} \geq 0$ on $\mathbb{R}$. If $y_{0} \geq 0$, then Lemma 3.7 ensures that $y \geq 0$ for all $t \in[0, T)$. By $u=p * y$ and the positivity of $p$, we infer that $u(t, \cdot) \geq 0$ for all $t \geq 0$. Note that

$$
u(t, x)=\frac{e^{-x}}{2} \int_{-\infty}^{x} e^{\xi} y(t, \xi) d \xi+\frac{e^{x}}{2} \int_{x}^{\infty} e^{-\xi} y(t, \xi) d \xi
$$

and

$$
u_{x}(t, x)=-\frac{e^{-x}}{2} \int_{-\infty}^{x} e^{\xi} y(t, \xi) d \xi+\frac{e^{x}}{2} \int_{x}^{\infty} e^{-\xi} y(t, \xi) d \xi
$$

From the above two equations, we deduce that

$$
\begin{aligned}
& u(t, x)+u_{x}(t, x)=e^{x} \int_{x}^{\infty} e^{-\xi} y(t, \xi) d \xi \\
& u(t, x)-u_{x}(t, x)=e^{-x} \int_{-\infty}^{x} e^{\xi} y(t, \xi) d \xi
\end{aligned}
$$

By $y \geq 0$ for all $t \in[0, T)$ and Lemma 3.5, we have

$$
\left|u_{x}(t, x)\right| \leq u(t, x) \leq \frac{\sqrt{2}}{2}\left\|u_{0}\right\|_{1}, \quad \forall(t, x) \in[0, T) \times \mathbb{R} .
$$

In the case when $y_{0}(x) \leq 0$ on $\mathbb{R}$, we can repeat the above proof to get the desired result. This completes the proof the theorem.

\section{Peakon solutions}

In this section we define strong solutions and weak solutions for Equation (1.1). We also prove that its peakon solutions are weak solutions.

Definition 4.1 ([22]). If $u \in C\left([0, T) ; H^{s}\right) \cap C^{1}\left([0, T) ; H^{s-1}\right)$ with $s>3 / 2$ is a solution to Equation (2.3), then $u(t, x)$ is called a strong solution to Equation (2.3) (or (1.1)).

Note that Equation (1.1) has the soliton waves with corner at its peak, discovered in [13]. Obviously, such solitons are not strong solutions to Equation (2.3). In order to provide a mathematical framework for the study of solitons, we define the notion of weak solutions to Equation (2.3). Let

$$
F(u)=u^{2} u_{x}+p *\left(3 u u_{x} u_{x x}+2 u_{x}^{3}+3 u^{2} u_{x}\right) .
$$


Then Equation (2.3) can be written as

$$
u_{t}+F(u)=0, \quad u(0, x)=u_{0} .
$$

Definition 4.2. Assume $u_{0} \in H^{s}, s \in\left[0, \frac{3}{2}\right]$. If $u(t, x) \in L_{\mathrm{loc}}^{\infty}\left([0, T) ; H^{s}\right)$ and satisfies

$$
\int_{0}^{T} \int_{\mathbb{R}}\left(u \varphi_{t}-F(u) \varphi\right) d x d t+\int_{\mathbb{R}} u_{0} \varphi(0, x) d x=0
$$

for all $\varphi \in C_{c}^{\infty}([0, T) \times \mathbb{R})$. Let $C_{c}^{\infty}([0, T) \times \mathbb{R})$ denote the space of all functions on $[0, T) \times \mathbb{R}$, which is restricted to $[0, T) \times \mathbb{R}$ is a smooth function on $\mathbb{R}^{2}$ with compact support contained in $(-T, T) \times \mathbb{R}$. Then $u(t, x)$ is called a weak solution to Equation (2.3). If $u(t, x)$ is a weak solution on $[0, T)$ for every $T>0$, then it is called a global weak solution to Equation (2.3) (or (1.1)).

Theorem 4.3. The peakon solitary

$$
u(t, x)= \pm \sqrt{c} e^{-\left|x-c t-x_{0}\right|}, \quad c>0, \quad x_{0}=\text { constant }
$$

is a global weak solution to Equation (2.3).

Moreover, $\forall T>0, u(t, x) \in L_{\mathrm{loc}}^{\infty}\left([0, T) ; H^{1}\right)$.

Proof. Since $x_{0}$ is constant, it is only to consider $u(t, x)=\sqrt{c} e^{-|x-c t|}$. Note that

$$
\begin{aligned}
& \int_{0}^{T} \int_{\mathbb{R}} u \varphi_{t}-F(u) \varphi d x d t+\int_{\mathbb{R}} u_{0} \varphi(0, x) d x \\
& =\int_{0}^{T} \int_{\mathbb{R}}\left(u_{t}+F(u)\right) \varphi d x d t \\
& =\int_{0}^{T} \int_{\mathbb{R}}\left(u_{t}+u^{2} u_{x}+p *\left(3 u u_{x} u_{x x}+2 u_{x}^{3}+3 u^{2} u_{x}\right)\right) \varphi d x d t .
\end{aligned}
$$

Since

$$
\left\{\begin{array}{l}
u_{t}=c^{\frac{3}{2}} e^{-|x-c t|} \operatorname{sgn}(x-c t)=(c u) \operatorname{sgn}(x-c t), \\
u_{x}=-\sqrt{c} e^{-|x-c t|} \operatorname{sgn}(x-c t)=-(u) \operatorname{sgn}(x-c t),
\end{array}\right.
$$

it follows that

$$
u_{t}+u^{2} u_{x}=\left(c u-u^{3}\right) \operatorname{sgn}(x-c t)
$$


On the other hand, in view of (4.3), we have

$$
\begin{aligned}
& p *\left(3 u u_{x} u_{x x}+2 u_{x}^{3}+3 u^{2} u_{x}\right) \\
& =\partial_{x} p *\left(\frac{3}{2} u u_{x}^{2}+u^{3}\right)+\frac{1}{2} p * u_{x}^{3} \\
& =\int_{\mathbb{R}} \partial_{x}\left(\frac{1}{2} e^{-|x-y|}\right)\left(\frac{3}{2} u u_{x}^{2}+u^{3}\right)(t, y) d y+\int_{\mathbb{R}} \frac{1}{4} e^{-|x-y|} u_{x}^{3}(t, y) d y \\
& =\int_{-\infty}^{x}-\frac{1}{2} e^{y-x}\left(\frac{3}{2} u u_{x}^{2}+u^{3}-\frac{1}{2} u_{x}^{3}\right)(t, y) d y \\
& \quad+\int_{x}^{\infty} \frac{1}{2} e^{x-y}\left(\frac{3}{2} u u_{x}^{2}+u^{3}+\frac{1}{2} u_{x}^{3}\right)(t, y) d y \\
& =\int_{-\infty}^{x}-\frac{1}{2} e^{y-x}\left(\frac{5}{2}+\frac{1}{2} \operatorname{sgn}(y-c t)\right) u^{3}(t, y) d y \\
& \quad+\int_{x}^{\infty} \frac{1}{2} e^{x-y}\left(\frac{5}{2}-\frac{1}{2} \operatorname{sgn}(y-c t)\right) u^{3}(t, y) d y .
\end{aligned}
$$

If $x<c t$. Using $u(t, x)=\sqrt{c} e^{-|x-c t|}$, we deduce from (4.5) that

$$
\begin{aligned}
& p *\left(3 u u_{x} u_{x x}+2 u_{x}^{3}+3 u^{2} u_{x}\right) \\
&= \int_{-\infty}^{x}-e^{y-x} u^{3}(t, y) d y+\int_{x}^{c t} \frac{3}{2} e^{x-y} u^{3}(t, y) d y \\
&+\int_{c t}^{\infty} e^{x-y} u^{3}(t, y) d y . \\
&= \int_{-\infty}^{x}-c^{\frac{3}{2}} e^{4 y-x-3 c t} d y+\int_{x}^{c t} \frac{3}{2} c^{\frac{3}{2}} e^{2 y+x-3 c t} d y \\
&+\int_{c t}^{\infty} c^{\frac{3}{2}} e^{x+3 c t-4 y} d y . \\
&=- \frac{1}{4} c^{\frac{3}{2}} e^{3(x-c t)}+\frac{3}{4} c^{\frac{3}{2}}\left(e^{x-c t}-e^{3(x-c t)}\right)+\frac{1}{4} c^{\frac{3}{2}} e^{x-c t} \\
&=-c^{\frac{3}{2}} e^{3(x-c t)}+c^{\frac{3}{2}} e^{x-c t}=c u-u^{3} .
\end{aligned}
$$

Similarly, if $x \geq c t$, we have

$$
p *\left(3 u u_{x} u_{x x}+2 u_{x}^{3}+3 u^{2} u_{x}\right)=-c u+u^{3} .
$$


In view of (4.6) and (4.7), we obtain

$$
p *\left(3 u u_{x} u_{x x}+2 u_{x}^{3}+3 u^{2} u_{x}\right)=-\left(c u-u^{3}\right) \operatorname{sgn}(x-c t) .
$$

Combining (4.2), (4.4), (4.8) with Definition 4.2, we deduce the desired result. This completes the proof of Theorem 4.3.

\section{References}

[1] H. Amann, "Ordinary Differential Equation", W. de Gruyter, Berlin, 1990.

[2] R. BeAls, D. SATtinger and J. SZMigielski, Multipeakons and a theorem of Stieltjes, Inverse Problems 15 (1999), 1-4.

[3] R. CAMASSA and D. Holm, An integrable shallow water equation with peaked solitons, Phys. Rev. Letters 71 (1993), 1661-1664.

[4] R. Camassa, D. Holm and J. Hyman, An integrable shallow water equation, Adv. Appl. Mech. 31 (1994), 1-33.

[5] G. M. Coclite, K. H. KARLSEN and N. H. Risebro, Numberical schemes for computing discontinuous solutions of the Degasperis-Procesi equation, IMA J. Numer. Anal. 28 (2008), 80-105.

[6] A. Constantin, On the scattering problem for the Camassa-Holm equation, Proc. R. Soc. London, Ser. A, Math. Phys. Eng. Sci. 457 (2001), 953-970.

[7] A. CONSTANTIN and J. ESCHER, Wave breaking for nonlinear nonlocal shallow water equation, Acta Math. 181 (1998), 229-243.

[8] A. CONSTANTIN and L. Molinet, The initial value problem for a generalized Boussinesq equation, Differential Integral Equations 15 (2002), 1061-1072.

[9] A. Degasperis, D. D. Holm and A. N. W. Hone, A new integrable equation with peakon solution, Theoret. Math. Phys. 133 (2002), 1463-1474.

[10] J. Dieudonne, "Foundations of Morden Analysis", Academic Press, New York, 1969.

[11] J. EscheR, Y. LIU and Z. YIN, Shock waves and blow-up phenomena for the periodic Degasperis-Procesi equation, Indiana Univ. Math. J. 56 (2007), 87-117.

[12] A. FOKAS and B. FUCHSSTEINER, Symplectic structures, their Bäcklund transformation and hereditary symmetries, Physica D 4 (1981), 47-66.

[13] A. N. W. HONE and J. WANG, Integrable peakon equations with cubic nonlinearity, J. Phys. A: Math. Theor. 41 (2008), 372002.

[14] T. KATO, Quasi-linear equation of evolution, with applications to partical differential equations, In: "Spectral Theorey and Differential Equation", Lecture Notes in Math., Vol. 488, Spring-Verlag, Berlin, 1975, 25-70.

[15] T. KATO, On the Korteweg-de Vries equation, Manuscripta Math. 28 (1979), 89-99.

[16] T. KATO, On the Cauchy problem for the generalized Korteweg-de Vries equation, In: "Studies in Applied Mathematics", Adv. Math. Suppl. Stu., Vol. 8, Academic Press, New York, 1983, 93-128.

[17] T. KATO and G. Ponce, Commutator estimation and the Euler and Navier-Stokes equation, Comm. Pure Appl. Math. 41 (1998) 891-907.

[18] H. LUNDMARK, Formation and dynamics of shock waves in the Degasperis-Procesi equation, J. Nonlinear Sci. 17 (2007), 169-198.

[19] V. S. Mikhailov and V. S. Novikov, Perturbative symmetry approach, J. Phys. A: Math. Gen. 35 (2002), 4775-4790.

[20] A. PAZY, "Semigroup of Linear Operators and Applications to Partial Differential Equations", Spring Verlag, New York, 1986. 
[21] V. S. Novikov, Generalizations of the Camassa-Holm equation, J. Phys. A: Math. Theor. 42 (2009), 342002.

[22] Z. YIN, On the Cauchy problem for an integrable equation with peakon solutins, Illinois J. Math. 47 (2003), 649-666.

[23] Z. YIN, Global weak solutions to a new periodic integrable equation with peakon solutions, J. Funct. Anal. 212 (2004), 182-194.

Department of Mathematics Sun Yat-sen University 510275 Guangzhou, China wx18758669@yahoo.com.cn mcsyzy@mail.sysu.edu.cn 